\title{
La gestión de la comunicación de crisis en las organizaciones. Efectos de la entropía en el caso BBVA-Villarejo
}

\author{
Krisi-komunikazioaren kudeaketa erakundeetan. \\ Entropiaren ondorioak BBVA-Villarejo kasuan \\ Crisis communication management. \\ Entropy effects concerning BBVA-Villarejo case
}

\author{
María Yolanda Martínez Solana ${ }^{1 \star}$, José Miguel Túñez-López ${ }^{2}$ \\ ${ }^{1}$ Universidad Complutense de Madrid, ${ }^{2}$ Universidad de Santiago de Compostela
}

\begin{abstract}
RESUMEN: El juez de la Audiencia Nacional Manuel García-Castellón imputó a la entidad financiera BBVA como persona jurídica en el denominado caso Villarejo, atribuyéndole presuntos delitos de cohecho, descubrimiento y revelación de secretos, entre otros. La Fiscalía coincide con los planteamientos hechos por el magistrado. La judicialización de la gestión realizada por los directivos del banco, encabezados por su expresidente, produjo un impacto en sectores políticos y económicos. La presente investigación aborda la génesis, la participación de los actores y su reflejo en los medios de comunicación a través del estudio de caso de la secuencia informativa por medio de fuentes referenciadas y de manera diacrónica. Queda reflejada la reacción que se produce cuando la realidad comunicada (escasa) se confronta con lo que se va conociendo por la multipolaridad de las fuentes informativas. Los conceptos de entropía y reputación se observan como predictores de desenlace.
\end{abstract}

PALABRAS CLAVE: Comunicación organizacional; Reputación; Comunicación de crisis; Entropía comunicativa; Gestión.

ABSTRACT: The judge of the Audiencia Nacional Manuel García-Castellón charged criminally BBVA as legal person in the socalled "Villarejo case», attributing the entity the alleged crimes of bribery, discovery and revelation of secrets, among others. The Prosecutor's Office agrees with the considerations made by the judge. The judicialization of the management performed by the bank executives, headed by former president, had significant impact on the political and economic sectors. In the present work, the background, the participation of the actors and media coverage are addressed through the case study of the informative sequence by means of relevant sources in a diachronic perspective. The reaction that happens when communication of reality (scarce) is confronted with what emerges from the multipolarity of the information sources is proved. The concepts of entropy and reputation are observed as predictors of the outcome.

KEYWORDS: Organizational communication; Reputation; Crisis communication; Communicative entropy; Management.

\footnotetext{
* Correspondencia a / Corresponding author: María Yolanda Martínez Solana. Facultad de Ciencias de la Información. Universidad Complutense de Madrid. Av. Complutense, 3 (28040 Madrid) - mymartinez@ccinf.ucm.es - https://orcid.org/0000-0001-9164-4634

Cómo citar / How to cite: Martínez Solana, María Yolanda; Túñez-López, José Miguel (2021). "La gestión de la comunicación de crisis en las organizaciones. Efectos de la entropía en el caso BBVA-Villarejon, Zer, 26(50), 147-165. (https://doi.org/10.1387/zer.22074).
}

Recibido: 01 octubre, 2020; aceptado: 12 enero, 2021.

ISSN 1137-1102 - elSSN 1989-631X / (C) 2021 UPV/EHU 


\section{Introducción y contexto}

¿Es necesario que exista un plan de comunicación en las organizaciones? Sin duda, la respuesta es sí. Tanto en su vertiente interna como en la externa. Como ejemplo baste con recordar que las organizaciones necesitan dar a conocer su existencia y su funcionamiento. Para ello precisan relacionarse con los medios y desconocen sus códigos de funcionamiento. Si, además, la organización se enfrenta a una crisis de comunicación será extremadamente difícil salir indemne de ella sin un plan de gestión preestablecido. No obstante, aún podemos comprobar que existen ocasiones en que organizaciones, instituciones, gobierno, etc. adoptan el silencio como forma de enfocar una crisis de comunicación que está horadando su organización, sin tener en cuenta que ese no decir nada es uno de los mayores errores que se pueden producir. Otro error que puede darse en comunicación organizacional es entender ésta como el predominio de la comunicación externa descuidando la interna, en este caso también se estará cercenando el sistema comunicativo, ya que la comunicación interna es la garantía de que la información fluya de forma efectiva.

El artículo estudia la secuencia de acontecimientos relacionados con la entidad financiera BBVA bajo la presidencia de Francisco González Rodríguez y sus relaciones con el excomisario del Cuerpo Nacional de Policía, José Manuel Villarejo Pérez. El magistrado de la Audiencia Nacional, Manuel García-Castellón ha imputado al BBVA como persona jurídica en el denominado "caso Villarejo» por presuntos delitos de cohecho, descubrimiento y revelación de secretos y corrupción en los negocios.

La Fiscalía Anticorrupción, en la misma línea, considera que «desde el $B B V A$ se produjeron contrataciones y pagos ilícitos a la mercantil Cenyt, cuyo titular era Villarejo, que afectaron a varias áreas sensibles del banco y a diversos ejecutivos de la entidad, durante un prolongado periodo temporal.» El espionaje masivo del Grupo Cenyt entre 2004 y 2005 estuvo encaminado a frustrar la toma de control del banco por la constructora Sacyr y José Manuel Villarejo Pérez percibió del BBVA (entre 2012 y 2017) cinco millones de euros. La eclosión de caso BBVA-Villarejo se produjo en mayo de 2018. Desde entonces la dilatación de la adopción de medidas por parte de la entidad bancaria ha derivado en una serie de consecuencias. La interacción entre política comunicativa y reputación son el objeto de este estudio.

\section{Comunicación de crisis}

Si existe un área en el que las crisis de comunicación han sido particularmente sensibles es la de la salud, además de que establece un marco de experiencias de referencia (Costa-Sánchez \& López-García, 2020; Castillo-Esparcia, Moreno \& Capriotti, 2020). Han pasado varias décadas desde que se produjeron varios falle- 
cimientos en los Estados Unidos de América por haber consumido el analgésico Tylenol (paracetamol) de la multinacional Johnson E Johnson. Al conocerse la muerte de siete personas en 1982 se desencadenó la alarma social. El laboratorio adoptó un sistema de gestión de la crisis que pivotó sobre la comunicación. En primer lugar, investigaron la forma en que se habían envenenado los fallecidos constatando que en las cápsulas del medicamento se había introducido cianuro; entonces se suspendió la producción del fármaco y se retiraron todos los lotes del producto que se habían distribuido en los Estados Unidos. Simultáneamente, Johnson E Johnson aplicó acciones de comunicación y relaciones públicas centradas en el diálogo y en la colaboración con la sociedad y con la Food and Drug Administration, la agencia de investigación criminal del Departamento de Justicia de Estados Unidos FBI y la policía estadounidense, para demostrar que el envenenamiento había sido un acto criminal al tiempo que difundían el mensaje de que no consumieran el medicamento. Johnson \& Johnson hizo una apuesta clara por la comunicación y antepuso la seguridad de la sociedad a los posibles efectos financieros de la crisis y asumió su responsabilidad frente a los consumidores, los médicos, sus propios empleados y accionistas. A la sociedad la mantuvo perfectamente informada y alerta a través de los medios de comunicación, difundiendo el mensaje de que no consumieran el medicamento.

Cuando concluyó la crisis se comprobó que la estrategia de la compañía farmacéutica había sido, además, rentable. JEJ no cayó en la tentación de negar cualquier relación de la compañía entre el consumo de Tylenol y los siete fallecimientos, y cooperó con los medios de comunicación. El resultado se vio recompensado. Kaplan (2012) en su artículo titulado "The Tylenol Crisis: How Effective Public Relations Saved Johnson \& Johnson", describía que la crisis del Tylenol había generado más de 125.000 noticias, lo que la sitúa en primer lugar en volumen informativo desde el asesinato del presidente John Fitzgerald Kennedy y constataba que sin la colaboración de los medios de comunicación JEJ no hubiera podido superar la crisis.

Pero en la fase previa a una crisis de comunicación, en la que aún es posible que no salga del lugar en que se ha generado (empresas, administraciones públicas, instituciones, etc.), también se puede gestionar de forma eficiente.

Según el Director Internacional de Gestión de Crisis de Edelman PR Worldwide, Mike Seymour (2000, 2004), aunque la realidad siempre es distinta, hay elementos que sí son iguales en todas las crisis: la necesidad de respuestas rápidas, ya que la aparición de la crisis es en segundos y su extensión como una mancha de aceite al universo de la empresa. Tendremos que hacer un esfuerzo para que la crisis quede dentro de la compañía y que el consumidor, por supuesto protegido, tenga la mayor información. Prevención, contar con especialistas y aplicar los resortes necesarios para que los medios puedan tener toda la información es la receta para gestionar bien una crisis, pues la crisis es una fase más por la que puede pasar cualquier empresa (Seymour, 2000). La falta de previsión, falta de transparencia informativa, falta 
de coordinación, falta de empatía o falta de agilidad en las respuestas han sido errores detectados en otros casos de gestión de comunicación de crisis previos (FernándezSouto, 2012; Oliveira; Huertas, 2018).

A pesar de que las empresas manifiestan que la comunicación forma parte de la toma decisiones por parte de los gestores, en 2014, último año del que existen datos, el número de empresas que disponían físicamente de un plan de comunicación integral, enlazado y relacionado con la estrategia general de negocio de la empresa era del 52 \%. (Fernández-Souto, Puentes-Rivera \& Vázquez-Gestal,2019). El porcentaje baja ostensiblemente en el caso de la Pequeña y Mediana Empresa (Costa-Sánchez, Túñez-López \& Míguez-González, 2020).

En un contexto de demanda de transparencia a todos los niveles y especialmente para las empresas que cotizan en bolsa (Briano \& Rodríguez, 2013), la gestión de la comunicación, y en clave más amplia, la de la empresa en general debería estar en manos de quienes sean capaces de generar una cultura corporativa que esté preparada para las crisis y que deben cumplir cinco condiciones (Agnesc, 2016):

1. Admitir que no son inmunes a las crisis

Deben tener claro que en esta época ninguna empresa es inmune a las crisis y que esta situación puede convertirse en una oportunidad para salir con éxito si están preparados. Las organizaciones más inteligentes son las que no son tímidas o asustadas para admitir esto, sino que optan por aprovechar esta realidad a su favor.

2. Identificar los escenarios de alto riesgo de su organización

Cada organización tiene una serie de escenarios de alto riesgo a los que son vulnerables, por lo cual identificarlos requiere un ejercicio de trabajo conjunto de los miembros del equipo ejecutivo. Éste es uno de los pasos más importantes y productivos en la implementación de una cultura corporativa lista para la crisis.

3. Desarrollar un modelo práctico de gobernanza de la gestión de crisis (con énfasis en la práctica). Para una gestión exitosa de la crisis en una organización tener como base un equipo fuerte en el que se tengan claras las funciones y responsabilidades. Un modelo de gobernanza fuerte refleja la estructura de gobierno de la organización, tiene roles claramente definidos y responsabilidades para cada miembro del equipo de gestión de crisis, e incluye protocolos internos de escalada que aseguran una escalada rápida cuando el tiempo es esencial.

4. Prevenir lo prevenible y prepararse para lo impredecible

Una vez definidos los escenarios de alto riesgo en la organización, los líderes deben tomarse el tiempo para analizar vulnerabilidades adicionales y prepararse para lo no previsible. Trabajan para desarrollar un programa de preparación para crisis que sea robusto, escalable y viable. 
5. Comprometerse a realizar ejercicios regulares.

Deben preparar a su equipo y sus habilidades para una gestión de crisis exitosa con la realización de simulaciones de crisis regulares y realistas. Los líderes fuertes que llevan a sus equipos al éxito de la gestión de crisis comprenden que la práctica hace que sea perfecta puesto que entienden que, aunque no quieren que su equipo tenga que enfrentarse a una crisis, cuando llegue el día inevitable, prefieren que su equipo tenga experiencia en gestión de crisis.

En realidad, la prevención de las situaciones de crisis tiene uno de sus principales pilares en la RSC. No resulta sorprendente, por tanto, que los ámbitos de actuación más publicitados por una empresa en materia de RSC tiendan a coincidir con los aspectos que pueden resultar más conflictivos en cada sector de actividad, es decir, con los más susceptibles de generar situaciones de crisis (Lalueza, 2014).

El departamento de comunicación que ha de gestionar la crisis debería estudiar y valorar, en primer lugar, cuáles son los focos y situaciones que pueden generarse en la propia empresa y en su entorno más inmediato (Oliveira \& Huertas, 2018). Seymour (2004) afirma que se debe definir cuál es el equipo más idóneo para poner en marcha estos tres principios: cómo vas a gestionar y resolver el problema; cómo vas a controlar la comunicación interna y la externa; y por último, asegurarse de que el resto del negocio no se vea afectado por la crisis.

La tarea del departamento de comunicación, por tanto, es definir todos los mensajes, trasladar a los ejecutivos y en especial a aquellos que van a actuar como portavoces, cuál es el papel que van a jugar en la crisis y ser extremadamente rápidos, tanto como lo es Internet.

\subsection{Reputación}

Si entendemos que la reputación empresarial es la percepción de los grupos de interés respecto a la actuación de la organización, como resultado del proceso histórico de sus interacciones, esta representa un recurso estratégico valioso, escaso y dificil de imitar. Por ello, el día a día de los gestores debería ir encaminado a intensificar las líneas de trabajo que construyan una reputación inmaculada (Álvarez-Gavilanes \& Murillo-Párraga, 2018).

La reputación es un proceso de valoración sobre la actuación que personas u organizaciones proyectan socialmente en relación a sus expectativas (Capriotti, 2009). Y en ese concepto se incluyen las acciones internas y externas. Es evidente que la comunicación juega un papel medular en la obtención de la reputación. 
Existe una relación directa entre la comunicación y la reputación empresarial, por tanto, es necesario adoptar un modelo de gestión de la comunicación pertinente con las exigencias del mercado (Álvarez-Gavilanes \& Murillo-Párraga, 2018) y las nuevas dinámicas sociales comunicativas (Alonso, 2016). Sin dejar de lado que los beneficios de una buena reputación empresarial son múltiples, entre ellos: la fidelización de los clientes, retención del mejor talento humano, satisfacción de clientes internos y externos, atracción de personal calificado, incremento de los ingresos, entre otros (Álvarez-Gavilanes \& Murillo-Párraga, 2018).

La reputación puede suponer para las organizaciones una clara ventaja competitiva frente a sus rivales y depende de su capacidad para gestionar un comportamiento corporativo, éticamente responsable, encaminado a satisfacer las necesidades reales y potenciales de los públicos que componen su universo (Fernández-Souto, AlmansaMartínez \& Castillo-Esparcia, 2018). En este proceso, es clave la adecuada gestión de la comunicación (Velarde-Ramos \& Pulido-Polo, 2018) y las actitudes honestas y conversacionales (Durántez-Stolle, 2018).

En la actualidad los principales agentes sociales del tejido empresarial europeo tienen una necesidad patente de incorporar la reputación como modelo de gestión y de cultura dentro de su organización. «De esta forma, generan relaciones sostenibles de confianza, credibilidad, compromiso y ética con sus públicos y su comunidad. Este nuevo modelo de gestión genera la necesidad de incorporar una formación específica de sus empleados para la implantación de la Reputación Corporativa como comportamiento corporativo en sus prácticas profesionales del día a día. Las compañías están incorporando la Reputación Corporativa como una competencia profesional necesaria que debe adquirir todo su personal» (Casado-Molina \& CuadradoMéndez, 2014: 6).

La pérdida de reputación puede afectar a cualquier empresa o grupo. Desafortunadamente para muchas empresas que se han ganado una gran reputación, el tan publicitado adagio "cuanto más grandes son, más dura es la caída» es cierto. Los stakeholders pueden perder la confianza incluso en las empresas más admiradas que les fallan (Gaines-Ross, 2008).

La reputación corporativa suele ir asociada a la de la cúpula de una organización. El valor del liderazgo en las organizaciones tiene múltiples facetas pero quizás la más sencilla identificación sea la de ser la cara de la empresa o institución (Cabrera \& Almansa-Martínez, 2016). Tal y como afirma Sotillo-Fraile (2010), el principal atractivo de la reputación del líder empresarial, en su consideración de activo intangible, es que se puede potenciar poniendo en práctica un proceso de gestión reputacional del liderazgo (GRL), que consiga impulsar la reputación del máximo ejecutivo y transferirla a la de la compañía. Se propone trabajar la reputa- 
ción del primer ejecutivo de la empresa y convertirla en una fuente de valor, con las siguientes premisas (Sotillo-Fraile, 2010):

1. Un buen líder es una garantía de futuro para la empresa y por ello su reputación forma parte de las expectativas sobre los resultados futuros de la misma.

2. La gestión reputacional del liderazgo (GRL) es un proceso mediante el cual el CEO guía y mejora su liderazgo trabajando sobre los aspectos de su gestión y personalidad que son más beneficiosos para la compañía. Más que un ejercicio de ego personal, la GRL es un ejercicio de responsabilidad del líder con la organización.

3. La utilidad de herramientas como los programas de gestión reputacional del liderazgo (GRL) reside en la identificación de aspectos a mejorar por parte del líder empresarial y del modo en que conviene mejorarlos, sirviendo habitualmente para identificar posibles mejoras en la propia compañía.

En una entrevista concedida al medio de comunicación El Pais, uno de los gurús de la comunicación, Wheeler (2004), manifestó que hay una «absoluta falta de confianza» en las empresas y los políticos y destacó el retraso en dar información como uno de los errores más habituales en comunicación. Y aconsejó: «Digan la verdad». O las compañías recuerdan que tienen que operar de forma ética y moral y tener en cuenta su responsabilidad social, o no sobrevivirán (Cebrián-Echarri, 2004).

\section{Metodología}

La metodología del presente trabajo se fundamenta en el estudio de caso. Investigaciones previas han incidido en la necesidad de fomentar los estudios de caso de crisis con un mayor enfoque hacia los contenidos y una cobertura más especializada (Smolak-Lozano \& García, 2020; Zurro-Antón, Moreno \& Fuentes-Lara, 2021). Inicialmente, se lleva a cabo un análisis de la secuencia informativa que se ha conocido sobre la relación entre Francisco González Rodríguez (exCEO del BBVA) y el excomisario José Manuel Villarejo Pérez (ya en prisión), utilizando fuentes solventes y con carácter diacrónico. La investigación ha avanzado para obtener resultados certeros sobre la repercusión de la gestión de una crisis derivada de un hecho delictivo y que afecta a uno de los mayores bancos españoles y el impacto social y reputacional por no aplicar un modelo de gestión de la crisis de comunicación subsiguiente.

El método aplicado ha conllevado:

A. Detección de la existencia de una gestión inadecuada de un problema medular para una entidad bancaria, estando inmersa en un presunto delito económico. 
B. Análisis de la secuencia informativa en el período ( pieza separada de la macrocausa Tándem, la número 9, se abrió el 4 de diciembre del año 2018 para investigar los contratos entre el BBVA y la empresa de Villarejo, hasta junio 2020) derivada del conocimiento de un problema que afecta a reputación y que permite intuir que la comunicación es el flanco más débil de BBVA para enfrentar socialmente la crisis.

C. Selección de los contenidos pertinentes para el campo de estudio.

D. Recopilación de los contenidos precisos para la investigación. Las bases de información y documentación han sido las siguientes:
D.1. Bibliográficas.
D.2. Hemerográficas.
D.3. Electrónicas.
D.4. Fuentes documentales de organismos oficiales.

E. Se ha procedido a la realización de un exhaustivo estudio de los acontecimientos producidos desde que se conocieron los primeros datos sobre la vinculación del excomisario José Manuel Villarejo Pérez al $B B V A$, septiembre de 2018, hasta agosto de 2019. Particular atención a las fechas en que se conoció la imputación de la entidad bancaria (julio 2019).

F. Elaboración de los resultados de la investigación y conclusiones.

El objetivo del presente seguimiento es el de extraer el aprendizaje relativo a los conceptos de comunicación de crisis y reputación, a partir del análisis de una situación de riesgo para la entidad bancaria española BBVA en el caso expuesto.

Se trata de una investigación innovadora y pertinente, dada la relevancia y repercusión pública de la organización y el trasfondo de la situación analizada.

\section{Resultados}

La comunicación es un proceso que se establece entre transmisor y receptor, que codifican y descodifican las señales que proceden de la fuente de información y son recibidas por un destinatario. Pero le dan al concepto "ruido» un factor de interferencia inherente al esquema de la comunicación y que es un factor de relevancia medular en el contenido final del mensaje (Shannon \& Weaver,1949). Esta interferencia inevitable en el proceso de la comunicación sin duda interfiere en la percepción del mensaje. A este concepto, se puede unir, aunque con carácter evitable, la entropía, que en las organizaciones mide el caos o la desorganización y en la información mide la incertidumbre de la fuente. ¿Qué sucede si hay entropía en la comunicación de las organizaciones? La entropía como materialización del desorden dependerá de nosotros o de nuestra organización. Cuanto menor sea, el tándem 
Ruido/Interferencia la gestión de la comunicación de la organización será más favorable.

El denominado «escándalo del BBVA», que ya se ha cobrado la imputación de ocho directivos y exdirectivos, la del propio banco como persona jurídica y que, muy posiblemente, a tenor del auto judicial, golpeará a toda su cúpula por la relación de la entidad con el excomisario José Manuel Villarejo Pérez y los servicios de espionaje que el príncipe de las cloacas le prestó hasta ayer mismo a cambio de una soldada millonaria es un ejemplo de esa entropía. Sorprende este mirar para otro lado porque estos casos de corrupción bancaria empiezan con una piedrecita rodando ladera abajo y terminan como una enorme bola que se lleva todo por delante y cuyos destrozos terminamos pagando todos a escote (Escudier-Vila, 2019).

La eclosión de este caso tiene su génesis en el temor de Francisco González Rodríguez a perder la presidencia de la entidad bancaria por el asalto accionarial de la constructora Sacyr en 2004. Para desbaratar la operación, la entidad recurrió a José Manuel Villarejo Pérez, que entonces seguía en la nómina de la Policía, y a sus métodos mafiosos para desactivarla, que incluían seguimientos y pinchazos telefónicos a unos una serie de objetivos. De ahí que las acusaciones de cohecho, revelación de secretos y corrupción en los negocios de los que el propio banco tendrá que responder como persona jurídica puedan impactar como un obús en su cuenta de resultados y disparar las alarmas del sistema financiero.

Los expertos en periodismo y en comunicación defienden que la línea de defensa del segundo Banco de España debería pasar por demostrar que el BBVA no recibió ningún beneficio — ni directo ni indirecto- a través de los pagos al excomisario José Manuel Villarejo Pérez. Toda vez que para exigir responsabilidades penales a una persona jurídica hay que demostrar que se benefició del hecho delictivo, según reza el artículo 31 bis del Código Penal. La Audiencia Nacional tiene en su poder facturación al grupo empresarial del excomisario José Manuel Villarejo Pérez del año 2017 y que sustenta el pago por parte del BBVA. La reacción de la entidad bancaria se ha circunscrito a la elaboración y difusión de un comunicado que su imputación por cohecho, revelación de secretos y corrupción entre particulares no supone la acusación formal por ningún delito (Díaz-Conde, 2019).

Tal y como se han desempeñado los dirigentes de la entidad financiera, no se han aportado las pruebas que generen confianza en la actuación del banco y se ha erosionado la confianza en el sector financiero. Según el profesor del programa de dirección en comunicación corporativa y management del IE Business School, López-Quesada (2017), la reputación corporativa es el activo intangible más valioso en la empresa y, ante una situación de crisis, la gestión eficaz de la comunicación contribuye a superarla antes y con menos consecuencias negativas en capitalización bursátil, retención del talento o preferencia del consumidor». Una buena gestión de la 
comunicación es vital para que la imagen de la compañía quede limpia o no sufra daños irreversibles. Más allá de sanciones económicas, de responsabilidades penales o del daño a la imagen, una crisis siempre hace referencia a sentimientos muy profundos, como el miedo, la inseguridad o la rabia. Las empresas deberían conocer cuáles son sus debilidades para así trazar un plan de prevención y gestión de crisis que les permita minimizar el impacto de una situación dificil que puede llevarla a perder la confianza de públicos clave, ya sean inversores, consumidores o empleados. Es necesario anticipar los peores escenarios y prepararse para ellos, entendiendo cuáles son las audiencias clave y qué mensajes debemos transmitirles.

López Quesada (2017) plantea la pregunta clave: ¿Qué se debe hacer antes de que estalle una crisis? Y asevera que no hay una estrategia exacta, pero sí existen diversos planes para prevenir esa situación, como realizar una auditoría de riesgos, tener un manual de crisis orientativo, monitorizar los issues que afectan al sector y la entidad en cuestión y tener un equipo experimentado o comité de crisis entrenado y formado con anterioridad al momento de la crisis. Si se habla de empresas cotizadas, los inversores quieren tener claro qué se va a hacer ante posibles situaciones de riesgos financieros, operacionales, estratégicos o de responsabilidad.

La preocupación por el impacto que supone la inexistencia de una gestión de la comunicación apropiada y su inexorable conversión en una crisis comunicativa ha tenido cumplida representación en las páginas de los medios informativos, durante los últimos meses. No solo en los géneros informativos o explicativos, sino en las páginas de Opinión. Así lo hace el editorial del 18 de marzo de 2019 del medio de comunicación El Pais titulado «Reputación bancaria: Las explicaciones del nuevo presidente del $B B V A$ a los accionistas no han despejado las dudas", en el que se indicaba que la reputación del $B B V A$ sigue en entredicho.

Juzgando los últimos movimientos de la cúpula de la entidad bancaria, ponía de manifiesto que «las explicaciones aportadas por el nuevo presidente, Carlos Torres Vila, en la junta general de accionistas celebrada el viernes pasado no han despejado las dudas acerca de las escuchas ilegales realizadas por el excomisario jubilado José Manuel Villarejo Pérez a políticos, empresarios y periodistas supuestamente contratadas durante la presidencia de su antecesor, Francisco González Rodríguez. Con independencia de las actuaciones en curso de la Audiencia Nacional, la investigación interna encargada por los responsables del banco debería ser suficientemente rigurosa y diligente para tratar de liberar cuanto antes la pesada hipoteca que pesa sobre uno de los más importantes bancos del país y su consejo de administración. Es necesario conocer de forma clara si esas escuchas fueron autorizadas por responsables del banco, si su financiación, dada la cuantía de las mismas, se sometió a los controles, contabilización y autorizaciones necesarias. Este no es un caso que pueda darse por zanjado con la renuncia provisional del anterior presidente a la presidencia de honor y de la fundación del propio banco, un día antes de la junta general, si las evidencias acaban 
validando comportamientos como los relatados en las últimas semanas. Sería, cuando menos, decepcionante que los ciudadanos, los clientes y los inversores contemplaran que la exhibición de valores éticos coexiste con prácticas absolutamente deplorables en el seno de organizaciones que deberían ser de las más respetables».

Es dificil obviar que hace poco que España ha salido de la crisis financiera y que existen secuelas de una importante erosión reputacional en todo el sistema bancario español, por lo que el rotativo apunta que «el asunto BBVA ha vuelto a dañar ese activo intangible que en la actividad bancaria es esencial, la confianza, el crédito de las instituciones. Por eso no ha de extrañar que el propio Banco Central Europeo, responsable último de la supervisión bancaria, haya mostrado su preocupación al respecto. Por lo mismo, sería conveniente que los supervisores españoles, Banco de España y CNMV, velaran por la amplitud y celeridad de las investigaciones abiertas en el propio banco, identificando si sus órganos de gobierno conocían o habían supervisado esas actuaciones, hoy bajo sospecha. Es una buena ocasión para fortalecer la necesaria confianza en el sistema bancario, en el sistema económico y las instituciones españolas, en definitiva».

Aunque la banca (como sector) reconoce sus problemas de reputación con una limitada autocrítica: «En parte lo merecemos» (Larrouy-Sánchez, 2018) lo cierto es que no solo se ha venido dando muestras de la desconfianza que han generado las entidades bancarias desde instancia sociales. En diciembre de 2018, el Banco de España avisó que el sector ha salido de la crisis con unos balances más fuertes, pero una imagen social más deteriorada, y situó este problema como uno de los desafios para la banca en los próximos años. El gobernador del Banco de España, en unas jornadas organizadas por la escuela de negocios IESE y la consultora EY manifestó que «la reputación se ha visto muy erosionada durante la crisis, debido a las malas prácticas del sector».

Como resultado de los acontecimientos que han afectado al sector durante la última crisis económica y a día de hoy, en particular, a Bankia y $B B V A$, la crisis institucional del sector bancario se ha ahondado en los dos últimos años y vuelven a copar portadas nacionales e internacionales.

El primer caso es el que más preocupa, ya que «muchos ciudadanos van a pensar que son actuaciones de la banca y no de una entidad concreta» (Giménez-Fernández, 2019). Audios e informes señalan que Francisco González Rodríguez, presidente de $B B V A$ durante 17 años -en los que el banco perdió un 14\% de su valor ajustado por dividendos y ampliaciones-, ordenó investigar la vida privada del exministro Miguel Sebastián Gascón y otros altos cargos del Gobierno de Zapatero al excomisario José Manuel Villarejo Pérez, en prisión preventiva desde noviembre de 2017 acusado de presuntos delitos de organización criminal, cohecho, blanqueo de capitales, extorsión y descubrimiento y revelación de secretos. El caso ya ha llegado al cuartel del supervisor europeo en Fráncfort. El Banco Central Europeo ha instado al 
consejo a que tome cartas en el asunto. Aunque se consideraba que aún no existían datos para valorar el impacto en el negocio del banco, y en bolsa continúa cotizando por debajo del valor en libros igual que antes de que explotara el escándalo, "ya hay advertencias, como la que ha hecho $S \in P$ : «Consideramos el daño reputacional en los «ratings» sí influye en el negocio y en el perfil financiero. En $B B V A$ hay una investigación y es pronto. Lo estamos monitoreando para ver si hay sanción o impacto», tal y como señaló Motti (2019), responsable del análisis de los bancos.

La evolución de los acontecimientos, intensificados tras la imputación por parte de la Audiencia Nacional ha permeado algunas sucursales del $B B V A$ en las que clientes y accionistas y los propios empleados se han contagiado la tensión y el nerviosismo ante la posibilidad de que el futuro del $B B V A$ pudiera ser «incierto». Los propios sindicatos, como $C C O O$, ya han pedido explicaciones a la actual cúpula del $B B V A$ sobre las consecuencias de la imputación del banco como persona jurídica (Marco, 2019).

El análisis de la situación por parte del sector económico critica al $B B V A$ los siguientes aspectos:

1. No romper con el pasado

No haberse desligado más clara y rápidamente de Francisco González Rodríguez. Los indicios del escándalo saltaron en mayo de 2018 y desde entonces se puso en marcha la investigación liderada por los equipos internos y Garrigues. Fuentes próximas a la entidad ligan este proceso al hecho de que Francisco González Rodríguez adelantara su salida a finales de 2018. Coincidiendo con ella, concedió una entrevista a El País(2018) en la que afirmaba que «ese tema (Villarejo) para mí no tiene relevancia» y «se han hecho las cosas como hay que hacerlas». Unos días después El Confidencial y Moncloa.com publicaban que el excomisario José Manuel Villarejo Pérez había pinchado 4.000 teléfonos para el banco.

2. Velocidad

En el sector financiero hay consenso de que esta crisis se podría haber resuelto en unos pocos meses, con una investigación a fondo liderada por los independientes del consejo, el consejero delegado y las firmas externas - Garrigues (bufete de cabecera del banco), PwC y Uría Menéndez-. El trabajo lo pilotaron inicialmente Arbizu; el exjefe de gabinete de Francisco González Rodríguez, Joaquín Gortari; y la directora de los servicios jurídicos, María Jesús Arribas.

El nuevo CEO del banco, Onur Genç, ha señalado que la investigación se ha hecho "en un tiempo récord», lo que dejó perplejo a más de uno. Aunque es dificil predecir qué hubiera pasado en otras circunstancias, en el mundo jurídico se repite que $B B V A$ ha perdido la iniciativa al no hacer el trabajo antes de que se abriera el caso judicial, lo que podría haberle permitido personarse como perjudicado en el caso en lugar de como imputado. 
3. Falta de alineación con los supervisores

Esta falta de agilidad es algo que le ha costado al banco reproches por parte del Banco Central Europeo, el Gobierno español y la $C N M V$, en más de una ocasión. La primera, cuando la entidad no se había todavía desligado de Francisco González Rodríguez a comienzos de año, y la segunda, más recientemente, tras seis meses de investigación interna de la mano de decenas de profesionales. El consejero delegado de la entidad bancaria (Genç) intentó contextualizar este punto, dado lo complicado que es mirar miles de correos electrónicos, mensajes, llamadas y documentos durante los últimos 14 años. Y, como ha recordado en innumerables ocasiones el actual Presidente de la entidad bancaria (Torres-Vila), su prioridad número uno ha sido colaborar con la Justicia.

4. No manejar el relato

Un posible cuarto error es haberse puesto de perfil durante los últimos meses, amparándose en el respeto a la investigación interna y judicial. La máxima del banco es no comentar la mayor parte de las informaciones que se publican, con lo que se pierde una oportunidad de controlar el mensaje. La entidad bancaria y el Banco Central Europeo están tranquilos dado que, más allá del reconocido impacto reputacional, este caso no ha impactado en el negocio, aunque hay una delgada línea entre que una crisis esté manejada y se descontrole.

Es precisamente la vertiente reputacional la que ha influido en la advertencia de la agencia de calificación Moody's al BBVA de que la imputación por el «caso Villarejo» expone al banco a un daño reputacional y podría, en última instancia, afectar negativamente a su solvencia. Así lo ha señalado la firma de rating en un comentario emitido con fecha 31 de julio tras conocerse la imputación del banco por parte del juez de la Audiencia Nacional, Manuel García-Castellón, por cohecho, corrupción y revelación de secretos en el conocido como "caso Villarejo». "Aunque es demasiado pronto para evaluar a qué acción del regulador, si la hubiera, podría enfrentarse el $B B V A$, la acusación expone al banco a daños en la reputación y, en última instancia, podría afectar negativamente a su solvencia» (eldiario.es, 2019). La calidad en la gobernanza es "particularmente importante» para los bancos, habida cuenta de que operan con un elevado apalancamiento y son generalmente más sensibles a la confianza que las empresas no financieras, particularmente teniendo en cuenta su esquema de financiación. "Las consecuencias de un fallo en el gobierno corporativo podrían ir más allá del efecto inmediato de una potencial multa. Un error en la gobernanza también distrae la atención del equipo directivo, así como los recursos, otro punto negativo para su perspectiva crediticia», apunta la agencia calificadora Moody's.

Recientemente, $B B V A$ ha creado un nuevo apartado de su web corporativa para recoger la información relativa al proceso judicial que investiga la relación del 
banco con el excomisario del Cuerpo Nacional de Policía José Manuel Villarejo Pérez a través de su empresa Grupo Cenyt. Por ese canal ha informado de que las líneas internas de investigación (la auditoría forensic que BBVA encargó a PwC, Garrigues y Uría Menéndez) ya han concluido. A renglón seguido informa que el banco «no puede compartir sus resultados, dado que se trata de un proceso judicializado» (Bolsamania, 2019). A pesar de ser el asunto que centra el interés del sector financiero, tanto nacional como internacional, los gestores del $B B V A$ únicamente incluyen unas entradas entre las que se encuentra el Blog interno del BBVA denominado ThePulse. En el momento de la imputación del banco, se han destinado 11 líneas al caso, firmadas por el presidente.

«No querría terminar sin hablar del caso Cenyt, que desafortunadamente para todos también ha tenido protagonismo estos meses. Os reitero, como dije en la carta que os envié en enero, mi firme compromiso con el esclarecimiento de los hechos y con el cumplimiento de la Ley. Nuestra investigación del asunto avanza a buen ritmo, aunque no puedo comentar nada porque, como sabéis, está supeditada a la investigación judicial, todavía bajo secreto de sumario. Como era de esperar, hemos visto también que se van dando pasos en esa causa judicial, con la que seguimos colaborando activamente. Mientras el asunto se resuelve tenemos que evitar prejuzgar hechos no constatados y, sobre todo, debemos mantener nuestro foco en nuestros clientes y trabajar conforme a nuestros valores para hacer realidad nuestro propósito y tener un impacto positivo en la sociedad» (Torres-Vila, 2019). El presidente del $B B V A$ se refiere al "firme compromiso con el esclarecimiento de los hechos», que realizó el pasado mes de enero, pero no comenta nada sobre la investigación interna, ocho meses después. El «caso Cenyt» ocupa las portadas de los diarios, y la entidad bancaria no informa ni a sus empleados, ni a los clientes.

El funcionamiento de los mecanismos de gestión de una crisis sobrevenida en una organización está ampliamente estudiado y analizado, más si cabe, en el actual mundo globalizado. Y, del mismo modo en que se conocen los procesos exitosos, sucede lo mismo con los fracasos. De esta forma, cualquier organización podrá ver cómo quiere que sea su devenir en situaciones de riesgo y crisis.

\section{Conclusiones}

El análisis de los documentos analizados en la investigación permite afirmar que la actitud de los responsables del $B B V A$, desde que salieron los primeros datos que relacionaban a José Manuel Villarejo Pérez (ya en prisión) con la entidad bancaria y que mostraban la comisión de delitos en que ambas partes estarían implicadas, fue la opuesta a lo que debería esperar de la entidad financiera: negar la realidad, no facilitar datos, no ser transparente, no colaborar con la administración de justicia y no adoptar medidas correctoras. 
De este modo se fue generando un clima de desconfianza entre los stakeholders y cuando el magistrado notifica la imputación del $B B V A$ se desencadena una crisis de comunicación, cuya repercusión se puede convertir en sistémica y, de duración, imprevisible. Al instaurarse una crisis de gestión y de comunicación se desencadenan una serie de acontecimientos con carácter inexorable: si la fuente informativa no facilita información a los medios, éstos buscarán otras vías encaminadas a seguir el rumbo de los acontecimientos, pues la noticia no deja de existir, aunque la fuente la niegue; simplemente saldrá con otra base documental (quizás otra fuente menos fiable, y más interesada) y se habrá perdido una ocasión de que la versión inicialmente solicitada por el periodista, salga a la luz pública (MartínezSolana, 2005).

La entropía comunicacional que se instala en el seno de una organización cuando la gestión de la crisis es la opuesta a la idónea, desemboca en un deterioro de la imagen y de la reputación empresarial, que no ha sido atendida por los actuales gestores del banco.

La repercusión en los medios de comunicación del caso BBVA-Villarejo ha reflejado la reacción que se produce cuando la realidad comunicada (escasa) se confronta con lo que se va conociendo por la multipolaridad de las fuentes informativas, sobre todo cuando la fuente es oficial y procede de los órganos de justicia.

Queda patente que existe un fracaso comunicativo por parte del $B B V A$, ligado a la actitud comunicativa que ha ido protagonizando durante el año y medio que dura la evolución del caso. Se podría considerar que ha sido el relato lo que ha fallado, sobre todo, pero quizás sería obviar que la realidad es que no ha existido tal, ya que esta entidad bancaria ha negado la existencia de irregularidad hasta que magistrado de la Audiencia Nacional, Manuel García-Castellón, ha imputado al BBVA como persona jurídica en el denominado «caso Villarejo» por presuntos delitos de cohecho, descubrimiento y revelación de secretos y corrupción en los negocios y la Fiscalía se ha manifestado en la misma línea.

Si analizamos la dinámica interna que se establece en una organización para gestionar la comunicación, sin realizarlo en función a la experiencia existente sobre el modelo a implantar, se produce inmediatamente una disfunción en el procedimiento o «entropía comunicacional» al generarse una disfunción dentro del proceso ya sea en forma de ruido o de un desorden entre lo que los medios y la sociedad demandan y lo que la organización $(B B V A)$ quiere aportar.

En ese punto, se desencadena una crisis de comunicación que requiere un plan de gestión, que marcará el futuro de la organización. De ahí la importancia de disponer de un plan de comunicación de crisis que permita una respuesta rápida y adecuada. 
Las entidades bancarias tienen en la confianza uno de sus principales activos. Sin confianza, su propia actividad empresarial carecería de sentido. Este tipo de crisis que se cronifican porque se alargan en el tiempo (todavía la entidad bancaria se encuentra en proceso judicial debido a los recursos y contrarecursos que se han ido presentando) van desgastando la credibilidad de la organización en el medio-largo plazo, mientras que una respuesta más ágil y certera por parte de la entidad habría detenido, si no el proceso judicial, sí la continuidad del proceso de erosión reputacional (Martínez-Solana, Fraçao \& Valarezzo, 2017).

Puesto que el caso no está todavía cerrado, esta investigación se mantiene en activo. Además, se recomienda el estudio de la gestión comunicativa de las entidades bancarias como línea de investigación a fortalecer en la agenda de investigación actual en España, ya que se trata de entidades de especial interés para el bienestar social, que hasta la fecha no han sido suficientemente estudiadas desde la gestión comunicativa.

\section{Contribución especíifca y orden de autoría en el artículo}

Marco teórico. Miguel Túñez-López. Yolanda Martínez-Solana. Metodología. Miguel Túñez-López. Yolanda Martínez-Solana. Resultados. Yolanda Martínez-Solana. Conclusiones. Yolanda Martínez-Solana. Redacción. Yolanda Martínez-Solana.

\section{Referencias bibliográficas}

Álvarez Gavilanes, J. E., \& Murillo Párraga, D. Y. (2018). Crisis de reputación empresarial en el entorno digital. Uniandes Episteme, 5(3), 194-209.

Alonso González, Marián (2016). Comunicación empresarial de las empresas del IBEX 35 en Twitter. Revista Mediterránea de Comunicación, 7(1), 137-161. Disponible en http:// mediterranea-comunicacion.org/.http://dx.doi.org/10.14198/MEDCOM2016.7.1.8

Briano Turrent, G., \& Rodríguez Ariza, L. (2013). Transparencia de la información corporativa en internet de las empresas del IBEX 35. Revista de contabilidad y dirección, 16, 187-208.

Cabrera Cabrera, M.; Almansa-Martínez, A. (2016). El director de relaciones públicas en las grandes empresas españolas/Public Relations Director in Spanish Companies. Revista internacional de relaciones públicas, 6(11), 113-134.

Capriotti, P. (2009). De la imagen a la reputación. Análisis de similitudes y diferencias. Razón y palabra, 14(70), 1-10.

Casado-Molina, A; Cuadrado-Méndez, F. (2014). La reputación corporativa: Un nuevo enfoque de las competencias transversales en el EEES. REDU Revista de Docencia Universitaria, Universidad Politécnica de Valencia, v. 12, n.1, pp. 353-371. Doi: 10.4995/ redu. 2014.6432 
Castillo Esparcia, A.; Moreno Fernández, A.; Capriotti, P. (2020). Relaciones públicas y comunicación institucional ante la crisis del COVID-19. Revista Internacional de Relaciones Públicas, 10(19), 1-6.

Cebrián-Echarri, B. (2004). Lo ocurrido en España abre nuevas formas de comunicación. El País, Madrid, 4 de abril de 2004, Entrevista: Adrian Wheeler (Experto de la Comunicación). https://elpais.com/diario/2004/04/04/sociedad/1081029602_850215.html

Costa-Sánchez, C., Túñez-López, J. M., \& Míguez-González, M. I. (2020). Gestión de la comunicación en la Pequeña y Mediana Empresa: potencial estratégico, filosofia y tendencia. Estudios sobre el Mensaje Periodístico, 26(3), 927-938. https://doi.org/10.5209/esmp.66244

Costa-Sánchez, C.; López-García, X. (2020). Comunicación y crisis del coronavirus en España. Primeras lecciones. El profesional de la información, v. 29, n. 3, e290304. https://doi.org/10.3145/epi.2020.may.04

Costa-Sánchez, C. (2017). Salas de comunicación online de las entidades bancarias en España. Entre el branded content informativo y el repositorio de notas de prensa. El profesional de la información (EPI), 26(4), 745-755. https://doi.org/10.3145/epi.2017.jul.18

Díaz-Conde, E. (2019). «El juez del “caso Villarejo” imputa al BBVA como persona jurídica». El Economista.es, 29 de Julio de 2019. https://www.eleconomista.es/banca-finanzas/ noticias/10017829/07/19/El-juez-del-caso-Villarejo-imputa-al-BBVA-como-personajuridica.html

Durántez-Stolle, P. (2017). Redes sociales virtuales en la comunicación corporativa del IBEX 35: usos, desarrollo e importancia según sus responsables. Revista Mediterránea de Comunicación/Mediterranean Journal of Communication, 8(1), 137-154. https://www.doi. org/10.14198/MEDCOM2017.8.1.10

El diario.es (2019). Moody's advierte de que la imputación de BBVA por el caso Villarejo podría afectar a su solvencia. eldiario.es, Sección Economía, 1 de agosto de 2019. https://www.eldiario.es/economia/Moodys-imputacion-BBVA-Villarejoafectando_0_926708065.html

El Pais (2019). REPUTACION BANCARIA: Las explicaciones del nuevo presidente del BBVA a los accionistas no han despejado las dudas. El País, Editorial del 18 de marzo de 2019. https://elpais.com/elpais/2019/03/17/opinion/1552835930_483726.html

Escudier-Vila, J. C.(2019). «La que has liao,pollito», Diario Público, 1 de agosto de 2019. https://blogs.publico.es/escudier/2019/08/01/la-que-has-liao-pollito/.

Fernández-Souto, A.; Puentes-Rivera, I.; Vázquez-Gestal, M. (2019). La gestión comunicativa de las grandes empresas en España: estructura, recursos y principales retos de sus responsables. Communication \& Society, Universidad de Navarra, v. 32, n. 1, pp. 161-176. doi: 10.15581/003.32.1.161-177

Fernández Souto, A. B., Almansa Martínez, A., Castillo Esparcia, A. (2018). Ética en las relaciones entre los medios de comunicación y los gabinetes de comunicación. Prisma social: revista de investigación social, (22), 1-19.

Fernández Souto, A. (2012). La comunicación y gestión de crisis. Notas comparativas entre el Caso Prestige y la tragedia del Golfo de México. Revista Anagramas, 10(21), 143-154. 
Gaines-Ross, L. (2008). Corporate reputation: 12 steps to safeguarding and recovering reputation. John Wiley \& Sons Ltd.

Giménez-Fernández, Ó. (2019). Los dos grandes bancos españoles, sumidos en una crisis institucional. El Confidencial, 17 de enero de 2019. https://www.elconfidencial.com/ empresas/2019-01-17/bbva-santander-reputacion-villarejo-orcel_1763522/

Larrouy Sánchez, D. (2018). La banca reconoce sus problemas de reputación con una limitada autocrítica: «En parte lo merecemos». El Diario.es, 12 de diciembre de 2018. https://www.eldiario.es/economia/reconoce-problemas-reputacion-autocriticamerecemos_0_845516349.html

Kaplan, T. (2012). The Tylenol Crisis: How Effective Public Relations Saved Johnson E Johnson. Pennsylvania: Pennsylvania State University.

https://studylib.net/doc/15994752/the-tylenol-crisis--how-effective-public-relationssaved-...

Lalueza, F. (2014). Responsabilidad social corporativa: las relaciones públicas profilácticas. In Túñez-López, M. \& Costa-Sánchez. Comunicación Corporativa: claves y escenarios (pp. 117-126). Barcelona: UOC.

López-Quesada, M. (2017). Cómo proteger la reputación en una crisis. IN SIGHTS IE University, https://www.ie.edu/insights/es/articulos/proteger-la-reputacion-una-crisis/

Marco, José Luis (2019). La tensión se traslada a las oficinas, clientes, empleados y accionistas del BBVA. Capital Madrid, 30 de julio de 2019. https://www.capitalmadrid. com/2019/7/30/53719/laq-tension-se-traslada-a-las-oficinas-clientes-empleados-yaccionistas-del-bbva.html

Martínez-Solana, Y. (2005). «Propuesta de un modelo responsable de información sanitaria: estudio del caso de la encefalopatía espongiforme bovina (EEB o «mal de las vacas locas»)». Tesis doctoral. Madrid: Universidad Complutense de Madrid. https://eprints.ucm.es/4666/

Martínez-Solana, Y.; Frazão-Nogueira, A.; Valarezo-González, K. (2017). Gestión de la comunicación de crisis en los atentados de 2017 en Reino Unido. Propuesta de un protocolo 2.0 para la comunicación de crisis. Revista Latina de Comunicación Social, 72, pp. 1.566 a 1.591. https://doi.org/10.4185/RLCS-2017-1235

Oliveira, A., \& Huertas Roig, A. (2018). La comunicación de crisis de Barcelona tras el atentado terrorista/The crisis communication of Barcelona after the terrorist attack. Revista Internacional de Relaciones Públicas, 8(15), 05-22.

Romero-Rodríguez, Luis Miguel; Torres-Toukoumidis, Ángel; Pérez-Rodríguez, María Amor (2017). Gestión Comunicacional de Crisis: Entre la agenda corporativa y mediática. Estudio de caso Volkswagen. Revista Internacional de Relaciones Públicas, v. 7, n. 13, pp. 83-100. Doi: 10.5783/RIRP13-2017-06-83-100

Ruiz-Sánchez, Belén; Esteban-Talaya, Águeda; Gutiérrez-Broncano, Santiago (2014). Reputación bancaria durante la crisis económica. Comparación entre las principales entidades financieras desde la perspectiva del cliente. Universia Business Review, n. 43, pp. 16-35. https://journals.ucjc.edu/ubr/article/view/909/1039

Seymour, M.; Moore, S. (2000). Effective Crisis Management: Worldwide Principles and Practice. London: Cassell. 
Seymour, M. (2004). Fighting On All Fronts. CEO Magazine, advertorial.

Shannon, C.; Weave, W. (1963). The Mathematical Theory of Communication». University of Illinois Press. https://pure.mpg.de/rest/items/item_2383164/component/ file_2383163/content

Smolak-Lozano, E., \& Ponce, D. G. (2020). La gestión de crisis online 2008-2018 y su tratamiento en áreas del conocimiento y la comunicación en España. Correspondencias $\mathcal{E}$ Analisis, (11), 6 .

Sotillo-Fraile, S. (2010). La reputación del CEO: un activo intangible clave para las empresas. Cuadernos de Gestión del Conocimiento Empresarial, n.21. https://www.bridgedworld. com/ftp/La_reputacion_del_CEO_CEDE.pdf http://www.directivoscede.com/sites/ default/files/document/conocimiento/31-07-2013/08cuaderno0000003800.pdf

Torres Vila, C. (2019). Bienvenidos a THE PULSE. BBVA, 22 de julio de 2019.https:// www.bbva.com/es/opinion/bienvenidos-a-the-pulse/

Túñez-López, M.; Costa-Sánchez, C.; Míguez, M.I. (2018). Avances y retos de la gestión de la comunicación en el siglo xxi. Procesos, necesidades y carencias en el ámbito institucional. Estudios sobre el mensaje periodístico, 2018, vol. 24, no

Velarde-Ramos, S. A.; Pulido-Polo, M. (2018). Reputación corporativa y gestión de la comunicación. Estudios Institucionales, v.V, n. 9, pp. 243-252.

Zuloaga-Montero, J. (2019). BBVA-Villarejo: manual de cómo no gestionar una crisis reputacional. El Confidencial, 3 de agosto de 2019. https://www.elconfidencial.com/ empresas/2019-08-03/bbva-villarejo-manual-como-no-gestionar-crisis_2159351/

Zurro-Antón, N., Moreno, M., \& Fuentes-Lara, M.-C. (2021). Comunicación de crisis (2008-2018). Revisión de los principales avances de conocimiento empírico en gestión de comunicación. Profesional de la Información, 30(1). https://doi.org/10.3145/ epi.2021.ene.07 